\title{
Uncertainty propagation in inverse reliability-based design of composite structures
}

\author{
Carlos Conceição António · Luísa N. Hoffbauer
}

\begin{abstract}
An approach for the analysis of uncertainty propagation in reliability-based design optimization of composite laminate structures is presented. Using the Uniform Design Method (UDM), a set of design points is generated over a domain centered on the mean reference values of the random variables. A methodology based on inverse optimal design of composite structures to achieve a specified reliability level is proposed, and the corresponding maximum load is outlined as a function of ply angle. Using the generated UDM design points as input/output patterns, an Artificial Neural Network (ANN) is developed based on an evolutionary learning process. Then, a Monte Carlo simulation using ANN development is performed to simulate the behavior of the critical Tsai number, structural reliability index, and their relative sensitivities as a function of the ply angle of laminates. The results are generated for uniformly distributed random variables on a domain centered on mean values. The statistical analysis of the results enables the study of the variability of the reliability index and its sensitivity relative to the ply angle. Numerical
\end{abstract}

examples showing the utility of the approach for robust design of angle-ply laminates are presented.

Keywords

Composite structures, Uncertainty propagation, Inverse RBDO, Uniform Design Method, Artificial Neural Network, Monte Carlo simulation, Reliability index variability, Relative sensitivities

\section{Introduction}

The most realistic failure analysis of structures under uncertainty is associated with the use of reliability analysis methods. Therefore, the need for reliability analysis associated with optimal design with respect to composite structures has increased in the last 15 years, and reliability-based design optimization (RBDO) of composite structures is currently a very important area of research (Adali et al. 2003; Boyer et al. 1997; Carbillet et al. 2009; António et al. 1996, 2001; Rais-Rohani and Singh 2004; Salas and Venkataraman 2009; Teters and Kregers 1997).

Approximate reliability methods, such as the first order (FORM) or second order (SORM) reliability methods, use the so-called most probable failure point (MPP) to estimate the failure probability (Melchers 1999). The applicability of approximate reliability methods depends on the number of uncertainty parameters involved and degree of nonlinearity of the system response. In the ladder case, it is necessary to 
use simulation techniques such as Monte Carlo simulation. Nevertheless, the efficiency of the method is poor when estimating low failure probabilities. To overcome this problem, advanced simulation techniques, such as importance sampling, have been considered. The use of approximate models in reliability analysis and RBDO has been studied. In particular, Artificial Neural Networks (ANNs) have been used to approximate the limit state function and its derivatives (Nguyen-Thien and Tran-Cong 1999; Deng et al. 2005). Cheng (2007) proposed a hybrid technique based on ANN in combination with genetic algorithms (GAs) for structural reliability analysis. The proposed ANN-GA method uses a back-propagation training algorithm for the ANN learning process, after which the GA searches the MPP point and corresponding reliability index. Cheng et al. (2008) propose another method for structural reliability analysis by integrating the Uniform Design Method (UDM) with ANN-based GA.

As a method of reliability analysis of structures, most of the aforementioned models use the ANN as an approximation model of the limit state functions as a way of reducing the computational effort. In this paper, a new approach based on an approximation model simulation calculated at the same time as the limit state function, reliability index and their derivatives is presented. The objective is to study the propagation of uncertainties of the input random variables, such as mechanical properties, on the response of composite laminate structures under an imposed reliability level. Robustness assessment of the reliability-based designed composite structures is considered and some criteria are outlined for the particular case of angle-ply laminates.

\section{Uncertainty propagation in RBDO}

The problem of uncertainty propagation in RBDO of composite laminate structures is studied. First, an approach based on the optimal design of composite structures to achieve a specified reliability level is proposed, and the corresponding maximum load is outlined as a function of ply angle. This corresponds to an inverse reliability problem performed for the mean values of the mechanical properties of composite laminates. Then, using the UDM, a set of design points is generated over a domain centered at mean values of random variables, aimed at studying the space variability. The Tsai number associated with the MPP, reliability index and sensitivities of the reliability index are obtained for each UDM design point, using the previously calculated maximum load as a reference. Second, using the generated UDM design points as input/output patterns, an ANN is developed based on supervised evolutionary learning. Third, using the developed ANN and a Monte Carlo procedure, the uncertainty propagation in structural reliability index is evaluated as a function of ply angle. Figure 1 shows the flowchart of the proposed approach.

The objective of the proposed approach is to study the propagation of uncertainties in input random variables, such as mechanical properties, on the response of composite laminate structures for a specified reliability level. The problem of uncertainty propagation in RBDO of composite laminate structures is addressed according to the following steps:

First step: An approach based on optimal design of composite structures to achieve a specified reliability level, $b_{\mathrm{a}}$, is considered, and the corresponding maximum load is calculated as a function of ply angle, $a$. This inverse reliability problem is solved for the mean reference values, $\beta_{\mathrm{i}}$, of mechanical properties of the composite laminates.

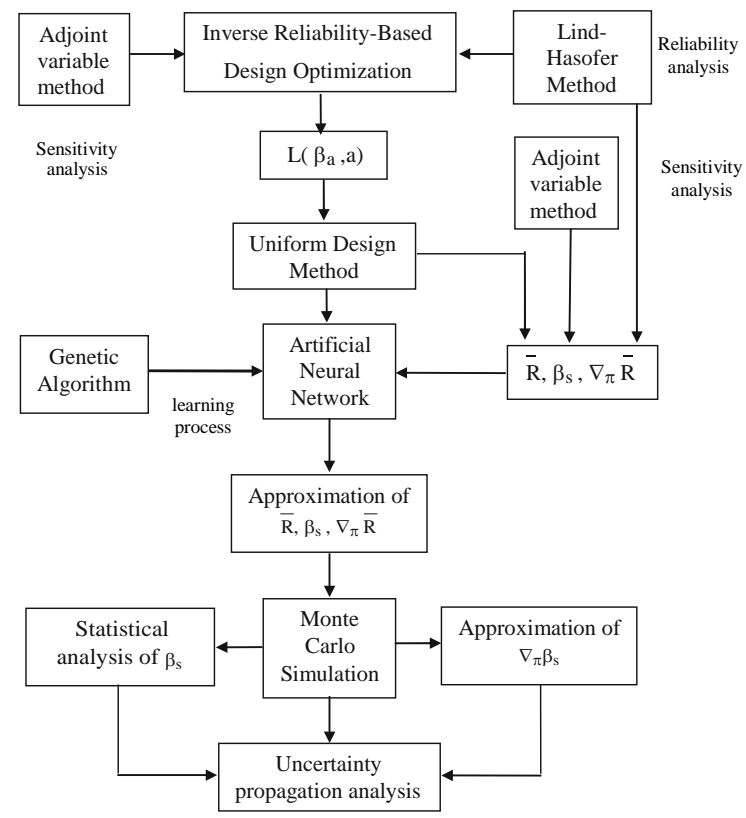

Fig. 1 Flowchart of proposed approach for uncertainty propagation analysis in RBDO 
Second step: Using the UDM, a set of design points belonging to the interval $\left.k_{\mathrm{i}}-a \beta_{\mathrm{i}} ; p_{\mathrm{i}} \mathrm{p} a p_{\mathrm{i}}\right]$ is generated, covering a domain centered at mean reference values of the random variables. This method enables a uniform exploration of the domain values necessary in the development of an ANN approximation model for variability study of the reliability index.

Third step: For each UDM design point, the Tsai number, $R$, associated with the MPP, structural reliability index, $b$, and their sensitivities, $\mathbf{r} b$ and $\mathbf{r} R$, are obtained using the previously calculated maximum load for mean values, $p_{\mathrm{i}}$, as a reference. The LindHasofer method is used for reliability index assessment (Hasofer and Lind 1974). The sensitivity analysis is performed by the adjoint variable method (António 1995; António et al. 1996).

Fourth step: An ANN is developed based on supervised evolutionary learning. The generated UDM design points and their calculated response values are used as input/output patterns.

Fifth step: Using the developed ANN and a Monte Carlo procedure, the variance of the structural reliability index is evaluated as a function of ply angle and uncertainty propagation is studied.

\section{Inverse reliability analysis}

The inverse reliability problem is solved for the mean values, $p_{i}$, of mechanical properties of composite laminates. An approach based on the design of composite structures to achieve a specified reliability level is proposed, and the corresponding maximum load is outlined. The objective function describing the performance of the composite structure is defined as the square difference between the structural reliability index, $b_{\mathrm{s}}$, and the prescribed reliability index, $b_{\mathrm{a}}$. The design variables are the ply angle, $a$, and load factor, $k$. The random variables are the elastic and strength material properties. Thus, the optimization problem is described as

$$
\begin{gathered}
\underset{\lambda, a}{\text { Minimise }}\left[\beta_{\mathrm{s}}\left(\lambda, a, \mu_{\pi}\right)-\beta_{\mathrm{a}}\right]^{2} \\
\text { Subject to: } \beta_{\mathrm{s}}(a, \pi) \geq \beta_{\mathrm{a}} \\
0 \leq a \leq \frac{\pi}{2}
\end{gathered}
$$

where $\mu_{\pi}$ is the realization of random variable $\pi$. The vector of applied loads is defined as $\mathbf{L}=\lambda \mathbf{L}^{\text {ref }}$, where
$\mathrm{L}^{\text {ref }}$ is the reference load vector. This is a conventional RBDO inverse optimization problem. To solve the inverse problem (1), a decomposition of the problem is considered. The Lind-Hasofer method and appropriate iterative scheme based on a gradient method are applied to evaluate the structural reliability index, $b_{\mathrm{s}}$, in the inner loop (Antó nio et al. 1996; António 1995). From the operational point of view, the reliability problem can be formulated as the constrained optimization problem

$$
\begin{array}{r}
\text { Minimize: } \beta(v)=\left(\mathbf{v}^{\mathrm{T}} \mathbf{v}\right)^{1 / 2} \\
\text { Subject to: } \varphi(\mathbf{v})=0
\end{array}
$$

where $\mathrm{v}$ is the vector of the standard normal variables, $b$ is the reliability index and $u \partial v p$ is the limit state function. The relationship between the standard normal variables and random variables is established using the following projection formula:

$$
v_{\mathrm{i}}=\frac{\pi_{\mathrm{i}}-\bar{\pi}_{\mathrm{i}}}{\sigma_{\pi_{\mathrm{i}}}}
$$

where $p_{\mathrm{i}}$ and $r_{p_{\mathrm{i}}}$ are, respectively, the mean values and standard deviations of the basic random variables. The limit state function that separates the design space into failure $(u(p) \backslash 0)$ and safe regions $(\varphi(\pi)>0)$ can be written as

$\varphi(\pi)=\bar{R}-1$

where $\bar{R}$ is the critical Tsai number, established as

$\bar{R}=\operatorname{Min}\left(R_{1}, \ldots, R_{k}, \ldots, R_{N_{x}}\right)$

and $N_{\mathrm{s}}$ the total number of points where the stress vector is evaluated. The Tsai number, $R_{k}$, which is a strength/stress ratio (Tsai 1987), is obtained from the Tsai-Wu interactive quadratic failure criterion and calculated at the $k$ th point of the structure solving equation

$1-\left(F_{i j} s_{i} s_{j}\right) R_{k}^{2}+\left(F_{i} s_{i}\right) R_{k}=0$

where $s_{i}$ are the components of the stress vector, and $F_{i j}$ and $F_{i}$ are the strength parameters associated with unidirectional reinforced laminate defined from the macro-mechanical perspective (Tsai 1987). The solu-

tion, $\mathrm{v}^{*}$, of the reliability problem in Eq. 2 is referred to, in technical literature, as the design point or MPP.

The bisection method used to estimate the load factor, $k$, is iteratively used in the external loop (António and Hoffbauer 2009). After the 
minimization of the objective function given in Eq. 1, the structural reliability index is $b_{\mathrm{s}} \& b_{\mathrm{a}}$ with some prescribed error, and the corresponding load vector is $\mathrm{L} \partial b_{\mathrm{a}} \mathrm{p}$.

\section{Uniform Design Method}

The purpose of the approximation methods is to reveal the relationship between response and input variables at the lowest cost. The key for this problem is to well-define a set of points that provide a good estimate of the expected output value. The KoksmaHlawka inequality (Fang et al. 1994; Fang and Wang 1994; Zhang et al. 1998; Liang et al. 2001) gives an error bound for the expected output value. This error

bound is equal to a measure of the variation of the response time discrepancy of the set of points over the entire domain. Using this inequality, the more uniform the points distributed over the range of input variables, the smaller the error. Therefore, points uniformly scattered in the domain are needed.

Obtaining points that are most uniformly scattered in the s-dimensional unit cube $C^{s}$ is the key of the UDM proposed by Fang et al. (1994), which is based on a quasi-Monte Carlo method. In fact, the UDM can be considered as a kind of experimental design with the aim of minimizing discrepancy. In this context, the discrepancy is used as a measure of uniformity that is universally accepted.

Let $P$ be a set of points on $C^{5}$. For any $\mathrm{g}=$ $\left(g_{1}, g_{2}, \ldots, g_{s}\right)$, let $N(\mathbf{g}, P)$ be the number of points in the hyper-cube $\left[0, g_{1}\right] \times\left[0, g_{2}\right] \times \cdots\left[0, g_{s}\right]$. The discrepancy of $P$ can be defined as

$\Psi(n, P)=\sup _{g \in C^{2}}\left|\frac{N(g, P)}{n}-g_{1} g_{2} \ldots g_{\mathrm{s}}\right|$

where $\frac{N(g, P)}{n}$ is the percentage of points falling in the hyper-cube $\left[0, g_{1}\right] \times\left[0, g_{2}\right] \times \cdots \times\left[0, g_{s}\right]$, with volume $g_{1} g_{2} \ldots g_{s}$ (Zhang et al. 1998). Here $g_{1} g_{2} \ldots g_{s}$ is also the percentage of volume occupied by $P$. Since the more uniformly distributed the set of points, the smaller the difference between $\frac{N(g, P)}{n}$ and $g_{1} g_{2} \ldots g_{s}, \Psi(n, P)$ is a good index. Fang et al. (1994) make use of good lattice points, glp, sets to construct a lot of tables of UDM. A UDM table is denoted by $U_{n}\left(q^{s}\right)$, where $U$ is the uniform design, $n$ the number of samples, $q$ the number of levels of each input variable, and $s$ the maximum number of columns of the table. For each UDM table, there is a corresponding accessory table, which includes a recommendation of columns with minimum discrepancy for a given number of input variables. Details of the algorithm for constructing a $U_{n}\left(n^{s}\right)$ table are given as follows:

- For a given $n$, find the set $H_{n}=\left\{h_{1}, h_{2}, \ldots h_{m}\right\}$ with m.d.c. $\left(n, h_{i}\right)=1$ and $h_{i} \leq n, i=1, \ldots, m$, with $m=\Phi(n)$, where $\Phi$ is the Euler function

$$
\Phi(n)=n\left(1-\frac{1}{p_{1}}\right)\left(1-\frac{1}{p_{2}}\right) \ldots\left(1-\frac{1}{p_{t}}\right),
$$

and $n=p_{1}^{r_{1}} p_{2}^{r_{2}} \ldots p_{t}^{r_{t}}$ is the prime decomposition of $n$.

- For any $s$ distinct elements of $H_{n}$, generate an $n \times s$ table where $u_{i j}=i h_{j}(\bmod n)($ for $i=1, \ldots, n$ and $j=1, \ldots, s)$ and $0<u_{i j} \leq n$, this is, $u_{i j}=n$ if $i h_{j}=k n$ for $k \in N$. There are $C_{s}^{m}$ possible tables. The one that has the small discrepancy is denoted by $U_{n}\left(n^{5}\right)$.

Finally, the UDM table must be transformed into a hyper-rectangle region corresponding to the input variable domain by linear transformation.

\section{ANN developments}

The adopted methodology, including the development of an ANN, is similar to the response surface method (RMS). The objective of the application of ANN is to overcome the difficulties associated with expensive assessment of the structural reliability for response variability study. Using the generated UDM design points as input/output patterns, an ANN is developed based on evolutionary learning. The developed based on evolutionary learning. The random variables are the input parameters and output parameters are the limit state function, reliability index and respective sensitivities.

\subsection{ANN topology definition}

The proposed ANN is organized into three layers of nodes (neurons): input, hidden and output layers. The linkages between input and hidden nodes and between hidden and output nodes are denoted by synapses. These are weighted connections that 
establish the relationship between input data $D_{i}^{\text {inp }}$ and output data $D_{j}^{\text {out }}$. In the developed ANN, the input data vector $D^{\text {inp }}$ is defined by a set of values for random variables $p$, which are the mechanical properties of composite laminates, such as elastic or strength properties. The longitudinal elastic modulus $E_{1}$; transversal elastic modulus $E_{2}$, transversal strength in tensile $Y$, and shear strength $S$ are considered the ANN input variables and denoted by $p$ $\left.1 / 4 k E_{1} ; E_{2} ; Y ; S\right]$. In this approach, each set of values for the random variable vector $p$ is selected using the UDM. The corresponding output data vector $\mathrm{D}^{\text {out }}$ contains the Tsai number, $R$, structural reliability index, $b_{\mathrm{s}}$, and Tsai number sensitivities. Figure 2 shows the topology of the ANN, showing the input and output parameters.

Each pattern, consisting of an input and output vector, needs to be normalized to avoid numerical error propagation during the ANN learning process. This is obtained using the following data normalization:

$$
\bar{D}_{k}=\left(D_{k}-D_{\min }\right) \frac{D_{N \max }-D_{N \min }}{D_{\max }-D_{\min }}+D_{N \min },
$$

where $D_{k}$ is the real value of the variable before normalization; and $D_{\min }$ and $D_{\max }$ are the minimum and maximum values of $D_{k}$, respectively, in the input/ output data set to be normalized. According to Eq. 8, the data set is normalized to values $\bar{D}_{k}$, verifying the conditions $D_{N \min } \leq \bar{D}_{k} \leq D_{N \max }$. Depending on the input or output data, different values of $D_{N \min }$ and $D_{N \max }$ can be used (0.1 and 0.9 , respectively, are the most common values). The sum of the modified signals (total activation) is performed through a function, designated as the Activation Function, $A(x)$. Thus, the activation of the $k$ th node of the hidden layer $(p=1)$ and output layer $(p=2)$ is obtained through sigmoid functions as follows:

$A_{k}^{(p)}=\frac{1}{1+e^{-\eta(p)} C_{k}^{(p)}}$

where $p$ represents the activation layer (either hidden or output layer) and $C_{k}^{(p)}$ are the components of the vector $\mathbf{C}^{(p)}$ given by

$\mathbf{C}^{(p)}=\mathbf{M}^{(p)} \mathbf{D}^{(p)}+\mathbf{r}^{(p)}$

where $\mathbf{M}^{(p)}$ is the matrix of the weights of synapses associated with the connections between input and hidden layers $(p=1)$ or between hidden and output layers $(p=2), \mathbf{r}^{(p)}$ is the biases vector on the hidden $(p=1)$ or output $(p=2)$ layers, $\mathbf{D}^{(p)}$ is the input data vector for the hidden $(p=1)$ or output $(p=2)$ layer. The scaling parameters $\eta^{(p)}$ influence the sensitivity of the sigmoid activation functions and must be controlled. The weights of the synapses, $m_{i j}^{(p)}$, and biases in the neurons at the hidden and output layers, $r_{k}^{(p)}$, are controlled during the leaming process.

\subsection{Evaluation of ANN performance}

The error between predefined output data and ANN simulated results is used to supervise the learning process, which is aimed at obtaining a complete model of the process. As a set of input data are introduced to the ANN, it adapts the weights of the synapses and values of the biases to produce
Fig. 2 Artificial Neural Network topology
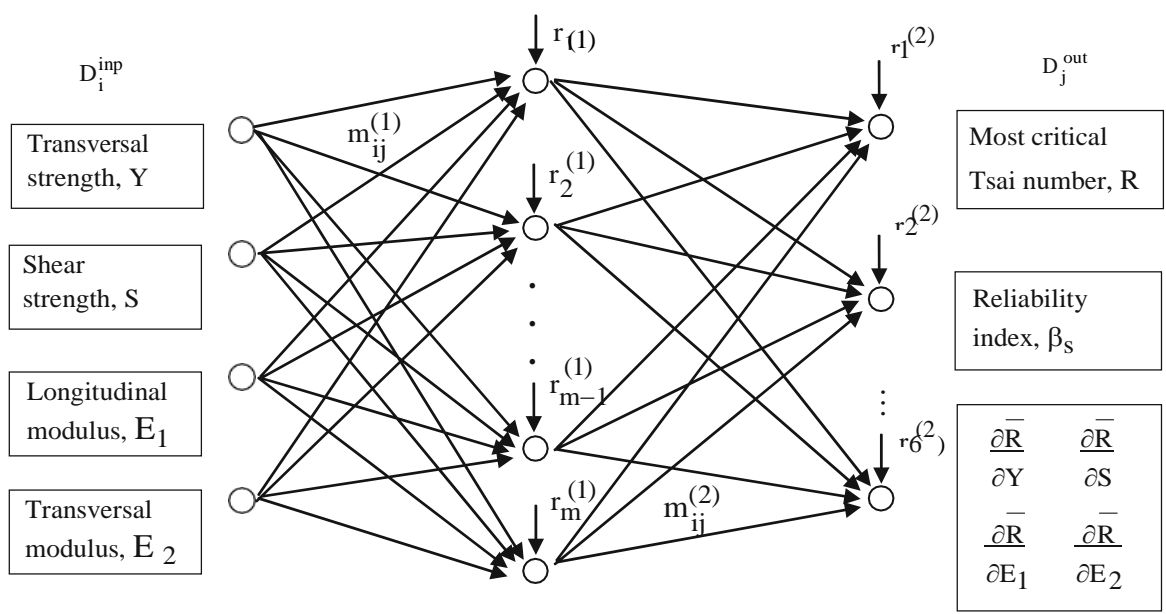
consistent simulated results through a process known as learning. For each set of input data and any configuration of the weight matrix $\mathrm{M}^{ð p \mathrm{p}}$ and biases $\mathbf{r}^{\delta p p}$, a set of output results is obtained. These simulated output results are compared with the predefined values to evaluate the difference (error), which is then minimized during the optimization procedure.

In general, the values of Tsai number at MPP and reliability indices are on the same magnitude order but very different from the magnitude order of the sensitivities. Therefore, a decomposition of the error is required and is defined as follows:

$$
\begin{aligned}
& E_{1}(\mathbf{M}, \mathbf{r})=\frac{1}{2 N_{\text {exp }}} \sum_{i=1}^{N_{\text {cop }}}\left(\left|\frac{\bar{R}^{\text {exp }}-\bar{R}^{\text {sim }}}{\bar{R}^{\text {exp }}}\right|+\left|\frac{\beta_{\mathrm{s}}^{\text {exp }}-\beta_{\mathrm{s}}^{\text {sim }}}{\beta_{\mathrm{s}}^{\exp }}\right|\right)_{i} \\
& E_{2}(\mathbf{M}, \mathbf{r})=\sum_{i=1}^{N_{\text {exp }}}\left(\left|\bar{R}^{\text {exp }}-\bar{R}^{\text {sim }}\right|+\left|\beta_{\mathrm{s}}^{\text {exp }}-\beta_{\mathrm{s}}^{\text {sim }}\right|\right)_{i} \\
& E_{3}(\mathbf{M}, \mathbf{r})=\sum_{i=1}^{N_{\text {eap }}} \sum_{j=1}^{N_{z}}\left(\left|\frac{\partial \bar{R}^{\exp }}{\partial \pi_{j}}-\frac{\partial \bar{R}^{\text {sim }}}{\partial \pi_{j}}\right|\right)_{i}
\end{aligned}
$$

where $N_{\exp }$ is the number of experiments considered in the set of design points of UDM and the superscripts sim and exp denote the simulated and experimental data, respectively. Equation 11 is the mean relative error and Eq. 12 is the absolute error. Both terms are associated with the most critical Tsai number and reliability index of the structural system. Equation 13 is the absolute error associated with the sensitivity relative to $N_{\pi}$ random variables. The influence of the biases of the neurons of the hidden and output layers is also included to stabilize the learning process:

$$
\begin{aligned}
\Gamma\left(\mathbf{r}^{(1)}, \mathbf{r}^{(2)}\right)= & \frac{1}{N^{\text {exp }}}\left(\sum _ { m = 1 } ^ { N ^ { \text { eq } } } \left[\frac{1}{N^{\text {hid }}} \sum_{k=1}^{N^{\text {hid }}}\left(r_{k}^{(1)}\right)^{2}\right.\right. \\
& \left.\left.+\frac{1}{N^{\text {out }}} \sum_{k=1}^{N^{\text {ese }}}\left(r_{k}^{(2)}\right)^{2}\right]\right)_{m}^{1 / 2}
\end{aligned}
$$

The errors obtained from Eqs. 11-13 and mean quadratic values of biases from Eq. 14 are reflected in the ANN learning. This means that the weights of the synapses and biases can be modified until the errors fall within a prescribed value. Therefore, the weight of the synapses in matrix $\mathrm{M}^{ð p p}$; and biases of the neurons of the hidden and output layers in vector $\mathbf{r}^{\delta p p}$; are modified to reduce the differences (supervised learning) throughout the optimization process.

\subsection{ANN Learning based on an evolutionary procedure}

The adopted supervised learning process of the ANN based on a GA uses the weights of synapses, $M^{ð p p}$; and biases of neural nodes at the hidden and output layers, $\mathbf{r}^{\tilde{p} p}$; as design variables. A binary code format is used for these variables. The number of digits of
each variable can be different depending on the

connection between the input-hidden layers or hidden-output layers. The bounds of the domain of the

den-output layers. The bounds of the domain of the learning variables and scaling parameters, $g^{(p)}$, are the control parameters.

The optimization problem formulation associated with the ANN learning process is based on the minimization of the errors defined in Eqs. 11-13 and bias values in Eq. 14. A regularization term associated with biases in the hidden and output neurons is included in the learning process and is aimed at stabilizing and accelerating the numerical procedure.

Since the objective of the evolutionary search is to maximize a global fitness function FIT associated with ANN performance, the optimization problem is defined as follows:

$$
\text { MaximizeFIT }=K-\left[\sum_{k=1}^{3} c_{k} E_{k}(\mathbf{M}, \mathbf{r})+c_{4} \Gamma\left(\mathbf{r}^{(1)}, \mathbf{r}^{(2)}\right)\right]
$$

where $K$ is an arbitrary constant used to obtain a

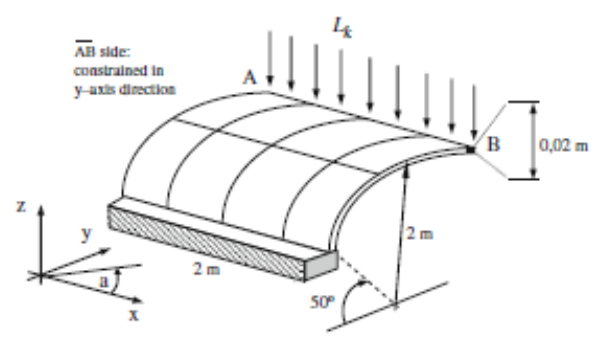

Fig. 3 FEM discretization and loading conditions of cylindrical composite shell 
Table 1 Mean reference values of mechanical properties of unidirectional composite layers

\begin{tabular}{llllr}
\hline Material & $E_{1}, \mathrm{GPa}$ & $E_{2}, \mathrm{GPa}$ & $E_{3}, \mathrm{GPa}$ & $\mathrm{m}$ \\
\hline E-glass/epoxy (Scotchply 1002) & 38.6 & 8.27 & 4.14 & 0.26 \\
\hline Material & $\mathrm{X}: \mathrm{X}^{0}, \mathrm{MPa}$ & $\mathrm{Y}: \mathrm{Y}^{0}, \mathrm{MPa}$ & $\mathrm{S}, \mathrm{MPa}$ & $q, \mathrm{~kg} / \mathrm{m}^{3}$ \\
\hline E-glass/epoxy (Scotchply 1002) & $1062 ; 610$ & $31 ; 118$ & 72 & 1600 \\
\hline
\end{tabular}

positive fitness and $c_{k}$ are weight constants of regularization.

A GA is an optimization technique based on the survival of the fittest and natural selection theory proposed by Charles Darwin. The GA basically performs on three parts: (1) coding and decoding random variables into strings; (2) evaluating the fitness of each solution string; and (3) applying genetic operators to generate the next generation of solution strings in a new population.

ı nree basic geneuc operators, nameıy seıection, crossover, and mutation are used in this paper. An elitist strategy based on conservation of the best-fit transfers the best-fitted solution into a new population for the next generation. Once the new population is created, the search process performed by the three genetic operators is repeated and the process continues until the average fitness of the elite group of the current generation now longer shows significant improvement over the previous generation. Further details on creating and using a GA for ANN learning can be found in António (2001).

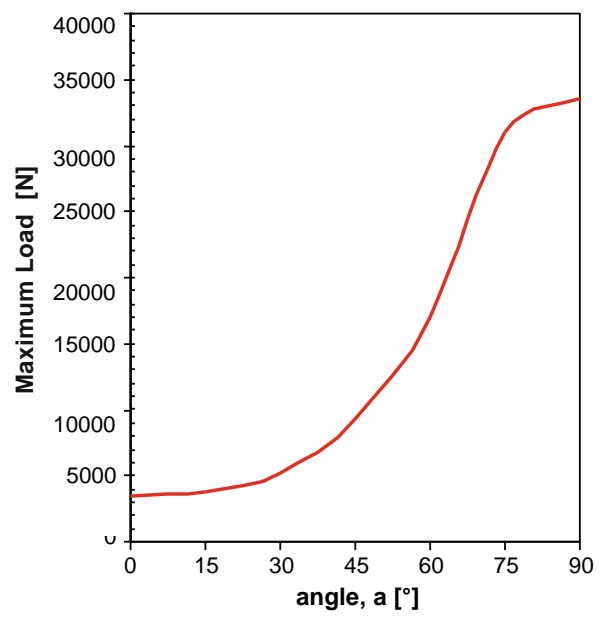

Fig. 4 Maximum load for $b_{\mathrm{a}}=3$, corresponding to the optimal solution of the inverse RBDO problem

\section{Numerical simulations}

To test the proposed approach applied to composite structures, a clamped cylindrical shell laminated structure is considered, as shown in Fig. 3. Nine vertical loads with mean value $L_{k}$ are applied along

Table 2 UDM design points for discrepancy W(n, $P)=0.1189$

\begin{tabular}{|c|c|c|c|c|}
\hline Design point & 1 & 4 & 6 & 9 \\
\hline 1 & 1 & 11 & 15 & 25 \\
\hline 2 & 2 & 22 & 2 & 22 \\
\hline 3 & 3 & 5 & 17 & 19 \\
\hline 4 & 4 & 16 & 4 & 16 \\
\hline 5 & 5 & 27 & 19 & 13 \\
\hline 6 & 6 & 10 & 6 & 10 \\
\hline 7 & 7 & 21 & 21 & 7 \\
\hline 8 & 8 & 4 & 8 & 4 \\
\hline 9 & 9 & 15 & 23 & 1 \\
\hline 10 & 10 & 26 & 10 & 26 \\
\hline 11 & 11 & 9 & 25 & 23 \\
\hline 12 & 12 & 20 & 12 & 20 \\
\hline 13 & 13 & 3 & 27 & 17 \\
\hline 14 & 14 & 14 & 14 & 14 \\
\hline 15 & 15 & 25 & 1 & 11 \\
\hline 16 & 16 & 8 & 16 & 8 \\
\hline 17 & 17 & 19 & 3 & 5 \\
\hline 18 & 18 & 2 & 18 & 2 \\
\hline 19 & 19 & 13 & 5 & 27 \\
\hline 20 & 20 & 24 & 20 & 24 \\
\hline 21 & 21 & 7 & 7 & 21 \\
\hline 22 & 22 & 18 & 22 & 18 \\
\hline 23 & 23 & 1 & 9 & 15 \\
\hline 24 & 24 & 12 & 24 & 12 \\
\hline 25 & 25 & 23 & 11 & 9 \\
\hline 26 & 26 & 7 & 26 & 6 \\
\hline 27 & 27 & 17 & 13 & 3 \\
\hline
\end{tabular}


the free linear side $(\mathrm{AB})$ of the structure. This free linear side $(\mathrm{AB})$ is constrained in the $y$-axis direction. The structure is made of one laminate. The balanced angle-ply laminates with eight layers and stacking sequence $1 / 2-a=\mathrm{p} \quad a=-a=\mathrm{p} a]_{\mathrm{S}}$ are considered in a symmetric construction. Ply angle, $a$, is referenced to the $x$-axis of the reference coordinate, as detailed in Fig. 3. All plies have a thicknesses of $2: 5 \times 10^{-3} \mathrm{~m}$.

The structural analysis of laminated composite structures is based on the finite element method (FEM) and shell finite element model developed by Ahmad (1969), and includes improvements from

Figueiras (1983). The Ahmad shell element is obtained from a 3D finite element using a degenerative procedure. It is an isoparametric element with eight nodes and five degrees of freedom per node, as described by Mindlin shell theory.
The laminate is made of an E-glass/epoxy composite system (Tsai 1987). The mean reference values of the elastic and strength properties of the ply material used in the laminate construction of the composite structure are presented in Table 1 . The elastic constants of the orthotropic ply are the longitudinal elastic modulus, $E_{1}$; transverse elastic modulus, $E_{2}$; in-plane shear modulus, $G_{12}$; out-of-plane shear modulus, $G_{13}$ and $G_{23}$; and in-plane Poisson's ratio, $m_{12}$. The ply strength properties are the longitudinal strength in tensile, $X$; longitudinal strength in compression, $X^{0}$; transverse strength in tensile, $Y$; transverse strength in compression, $Y^{\mathrm{O}}$; and shear strength, $S$.

To assess reliability, the longitudinal elastic modulus, $E_{1}$; transverse elastic modulus, $E_{2}$; transverse strength in tensile, $Y$; and shear strength, $S$; are the considered random variables and denoted by
Table 3 Input experimental values used in the ANN learning process

\begin{tabular}{|c|c|c|c|}
\hline$E_{1}$ & $E_{2}$ & $Y$ & $S$ \\
\hline $3.628400 \mathrm{E} ? 10$ & $8.155492 \mathrm{E} ? 09$ & $3.114308 \mathrm{E} ? 07$ & $7.565538 \mathrm{E} ? 07$ \\
\hline $3.646215 \mathrm{E} ? 10$ & 8.575354E ? 09 & 2.928308E ? 07 & $7.465846 \mathrm{E} ? 07$ \\
\hline 3.664031E ? 10 & 7.926477E ? 09 & $3.142923 \mathrm{E} ? 07$ & 7.366154E? 07 \\
\hline $3.681846 \mathrm{E} ? 10$ & $8.346338 \mathrm{E} ? 09$ & 2.956923E ? 07 & $7.266462 \mathrm{E} ? 07$ \\
\hline $3.699662 \mathrm{E} ? 10$ & 8.766200E ? 09 & $3.171538 \mathrm{E} ? 07$ & 7.166769E?07 \\
\hline $3.717477 \mathrm{E} ? 10$ & 8.117323E ? 09 & 2.985538E ? 07 & 7.067077E? 07 \\
\hline $3.735292 \mathrm{E} ? 10$ & $8.537185 \mathrm{E} ? 09$ & $3.200154 \mathrm{E} ? 07$ & $6.967385 \mathrm{E} ? 07$ \\
\hline $3.753108 \mathrm{E} ? 10$ & 7.888308E ? 09 & $3.014154 \mathrm{E} ? 07$ & 6.867692E?07 \\
\hline $3.770923 \mathrm{E} ? 10$ & 8.308169E ? 09 & $3.228769 \mathrm{E} ? 07$ & $6.768000 \mathrm{E} ? 07$ \\
\hline $3.788738 \mathrm{E} ? 10$ & 8.728031E? 09 & $3.042769 \mathrm{E} ? 07$ & 7.598769E? 07 \\
\hline $3.806554 \mathrm{E} ? 10$ & $8.079154 \mathrm{E} ? 09$ & $3.257385 \mathrm{E} ? 07$ & 7.499077E? 07 \\
\hline 3.824369E ? 10 & $8.499015 \mathrm{E} ? 09$ & $3.071385 \mathrm{E} ? 07$ & 7.399385E? 07 \\
\hline $3.842185 \mathrm{E} ? 10$ & 7.850138E ? 09 & $3.286000 \mathrm{E} ? 07$ & 7.299692E?07 \\
\hline $3.860000 \mathrm{E} ? 10$ & $8.270000 \mathrm{E} ? 09$ & $3.100000 \mathrm{E} ? 07$ & 7.200000E? 07 \\
\hline $3.877815 \mathrm{E} ? 10$ & 8.689862E ? 09 & 2.914000E ? 07 & $7.100308 \mathrm{E} ? 07$ \\
\hline 3.895631E ? 10 & $8.040985 \mathrm{E} ? 09$ & $3.128615 \mathrm{E} ? 07$ & 7.000615E? 07 \\
\hline $3.913446 \mathrm{E} ? 10$ & $8.460846 \mathrm{E} ? 09$ & $2.942615 \mathrm{E} ? 07$ & 6.900923E? 07 \\
\hline $3.931262 \mathrm{E} ? 10$ & 7.811969E ? 09 & $3.157231 \mathrm{E} ? 07$ & $6.801231 \mathrm{E} ? 07$ \\
\hline 3.949077E ? 10 & $8.231831 \mathrm{E} ? 09$ & $2.971231 \mathrm{E} ? 07$ & 7.632000E? 07 \\
\hline $3.966892 \mathrm{E} ? 10$ & $8.651692 \mathrm{E} ? 09$ & $3.185846 \mathrm{E} ? 07$ & $7.532308 \mathrm{E} ? 07$ \\
\hline 3.984708E ? 10 & $8.002815 \mathrm{E} ? 09$ & $2.999846 \mathrm{E} ? 07$ & 7.432615E?07 \\
\hline $4.002523 \mathrm{E} ? 10$ & 8.422677E ? 09 & $3.214462 \mathrm{E} ? 07$ & 7.332923E? 07 \\
\hline 4.020338E ? 10 & 7.773800E ? 09 & $3.028462 \mathrm{E} ? 07$ & 7.233231E? 07 \\
\hline $4.038154 \mathrm{E} ? 10$ & $8.193662 \mathrm{E} ? 09$ & $3.243077 \mathrm{E} ? 07$ & $7.133538 \mathrm{E} ? 07$ \\
\hline 4.055969E ? 10 & 8.613523E ? 09 & $3.057077 \mathrm{E} ? 07$ & 7.033846E? 07 \\
\hline $4.073785 \mathrm{E} ? 10$ & 7.964646E ? 09 & $3.271692 \mathrm{E} ? 07$ & $6.934154 \mathrm{E} ? 07$ \\
\hline 4.091600E ? 10 & 8.384508E ? 09 & $3.085692 \mathrm{E} ? 07$ & 6.834462E? 07 \\
\hline
\end{tabular}


$\left.\mathrm{p} 1 / 4 / 2 E_{1} ; E_{2} ; Y ; S\right]$. All random variables are noncorrelated, and follow a normal probability distribution function defined by their respective mean and standard deviation. The present study can be further extended to other random variables.

\subsection{Maximum reference load calculation}

To obtain the maximum reference load, the inverse RBDO problem defined in Eq. 1 is solved. The structural reliability index is $b_{\mathrm{s}} \& b_{\mathrm{a}}$ with some prescribed error, and the corresponding maximum load vector, $\mathrm{L} \partial b_{\mathrm{a}} \mathrm{p}$, can be obtained. The reliability assessment follows the procedure described in Eqs. 2-6. A prescribed reliability index $b_{\mathrm{a}}=3$ for the composite structure is considered. The mean values of the mechanical properties are assumed to be random variables and are defined in Table 1 , and the coefficient of variation of each random variable is set to $C V(p)=6 \%$, relative to the mean value.

The MPP values are obtained based on the LindHasofer method. After obtaining these values, the inverse RBDO, formulated in Eq. 1, is solved for $b_{\mathrm{a}}=3$ and the maximum load is outlined depending on ply angle $a$. The corresponding maximum load is plotted as a function of ply angle $a$, and shown in Fig. 4. This load is used as the reference load for further uncertainty propagation analysis in the ANNbased UDM and GA developments.

\subsection{ANN-based UDM and GA developments}

The UDM points are considered as experimental input values to be used in the ANN learning procedure. A number of 27 training data sets is selected inside the interval $h p_{\mathrm{i}}-0: 06 p_{\mathrm{i}} ; p_{\mathrm{i}}$ | 0:06 $p_{\mathrm{i}}$ ], with mean reference value $\beta_{\mathrm{i}}$ set as a random variable for each mechanical property and defined in Table 1. The UDM values are selected according to the approach proposed by Cheng et al. (2008).

After selecting table $U_{27}\left(27^{10}\right)$ of the UDM (Cheng et al. 2008), where columns 1, 4, 6, and 9 must be selected according to the respective accessory table for four variables and discrepancy $\mathrm{W}(n, P)=0.1189$, the resulting integer code format is presented in Table 2 . Then, the interval $k p_{\mathrm{i}}-0: 06 p_{\mathrm{i}} ; p_{\mathrm{i}} \mathrm{p} 0: 06 p_{\mathrm{i}}$ ] is equally discretized with 27 points and, using the integer code format from Table 2, the actual composition for $\mathrm{p} 1 / 4$ $\left.E_{1} ; E_{2} ; Y ; S\right]$ is obtained, as shown in Table 3.
Reliability analysis is performed for the input values from Table 3, and 27 input/output patterns are obtained and used in ANN development. For each UDM design point, the most critical Tsai number, $R$, associated with the MPP; reliability index of structure, $b_{\mathrm{s}}$; and sensitivities are obtained by using the maximum load previously calculated for each angle, $a$, as a reference and solving the inverse RBDO formulation of Eq. 1. A fixed standard deviation $r_{p_{\mathrm{i}}}{ }^{1 / 4}$ $0: 06 p_{\mathrm{i}}$ is used in the reliability index evaluation for all UDM design points, based on Lind-Hasofer method. The sensitivities are calculated based on the adjoint variable method.

A number of 10 neurons are considered for the hidden layer of the ANN topology. The ANN learning process is formulated as an optimization problem, defined in Eq. 15, with 116 design variables corresponding to 100 weights of synapses and 16 biases of neural nodes.

The ANN-based GA learning process is performed using a population of 21 individuals/solutions. The elite and mutation groups have seven and four solutions, respectively (António 2001). The binary code format with five digits is adopted for both designing the values of the weights of synapses, $M^{ð p p}$, and biases of neural nodes at the hidden and output layers, $\mathbf{r}^{\delta p p}$. The learning process is concluded after

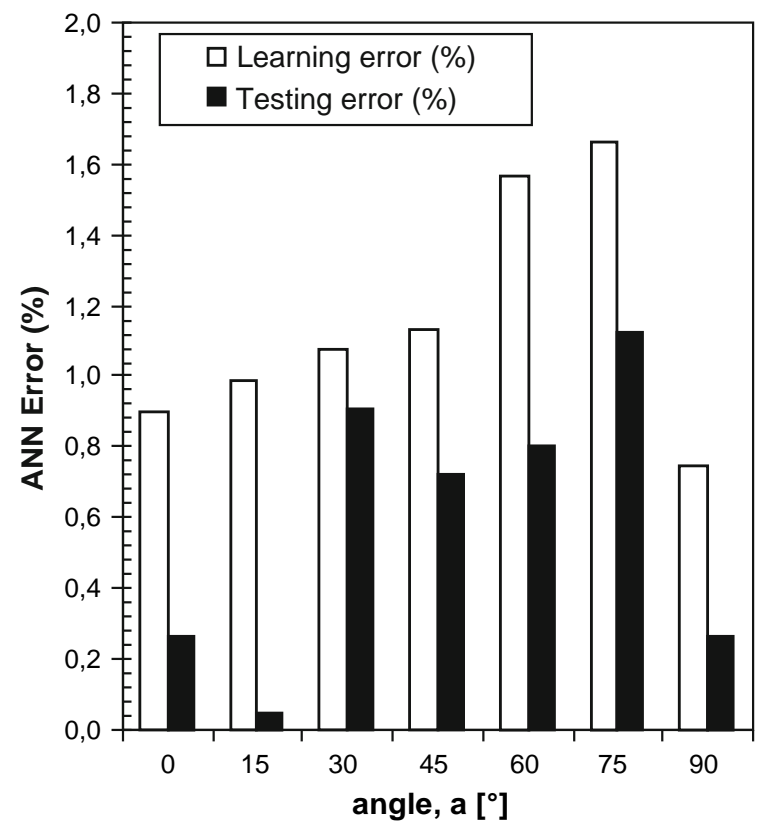

Fig. 5 Learning and testing relative errors for optimal ANNbased GA 
15,000 generations of the GA. The mean values in Table 1 (point 14 of UDM Table 2) are used for ANN testing. Figure 5 shows the relative errors in learning and testing processes corresponding to the optimal ANN.

\subsection{ANN-based Monte Carlo simulation}

The Monte Carlo Simulation (MCS) method is used to study the reliability index variability relative to uncertainties in the mean reference values of mechanical properties of composite structures. Using the
Table 4 Linear regression of data obtained using ANN-based MCS

\begin{tabular}{lll}
\hline Angle, $a(0)$ & $\begin{array}{l}\text { Linear } \\
\text { regression }\end{array}$ & $\begin{array}{l}\text { Coefficient of } \\
\text { determination }(\%)\end{array}$ \\
\hline 0 & $b=13.88 \mathrm{R}-13.96$ & 99.70 \\
15 & $b=13.10 \mathrm{R}-13.04$ & 95.33 \\
30 & $b=12.21 \mathrm{R}-12.21$ & 96.89 \\
45 & $b=8.65 \mathrm{R}-8.27$ & 98.10 \\
60 & $b=7.91 \mathrm{R}-7.61$ & 95.69 \\
75 & $b=46.54 \mathrm{R}-47.94$ & 96.97 \\
90 & $b=28.99 \mathrm{R}-28.98$ & 94.68 \\
\hline
\end{tabular}
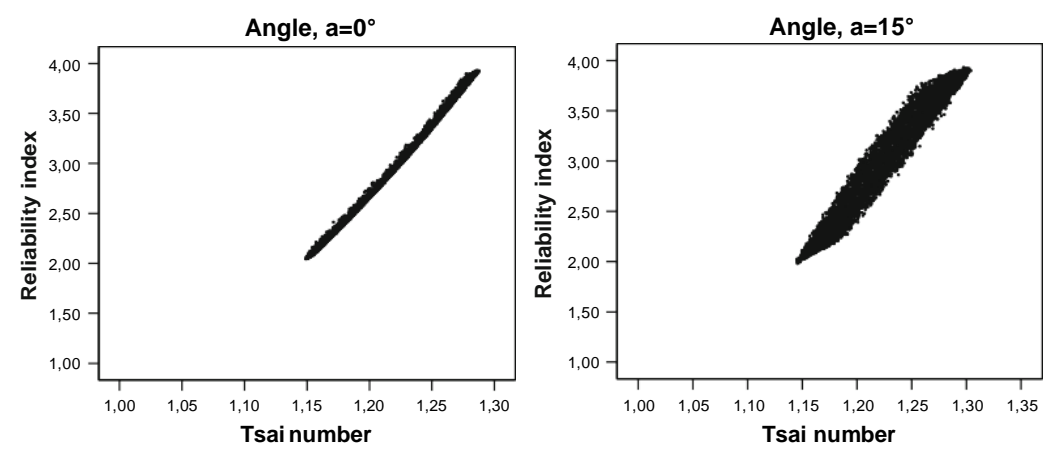

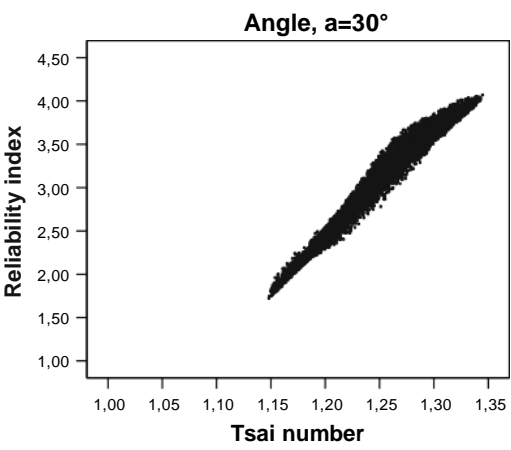

Angle, $a=30^{\circ}$
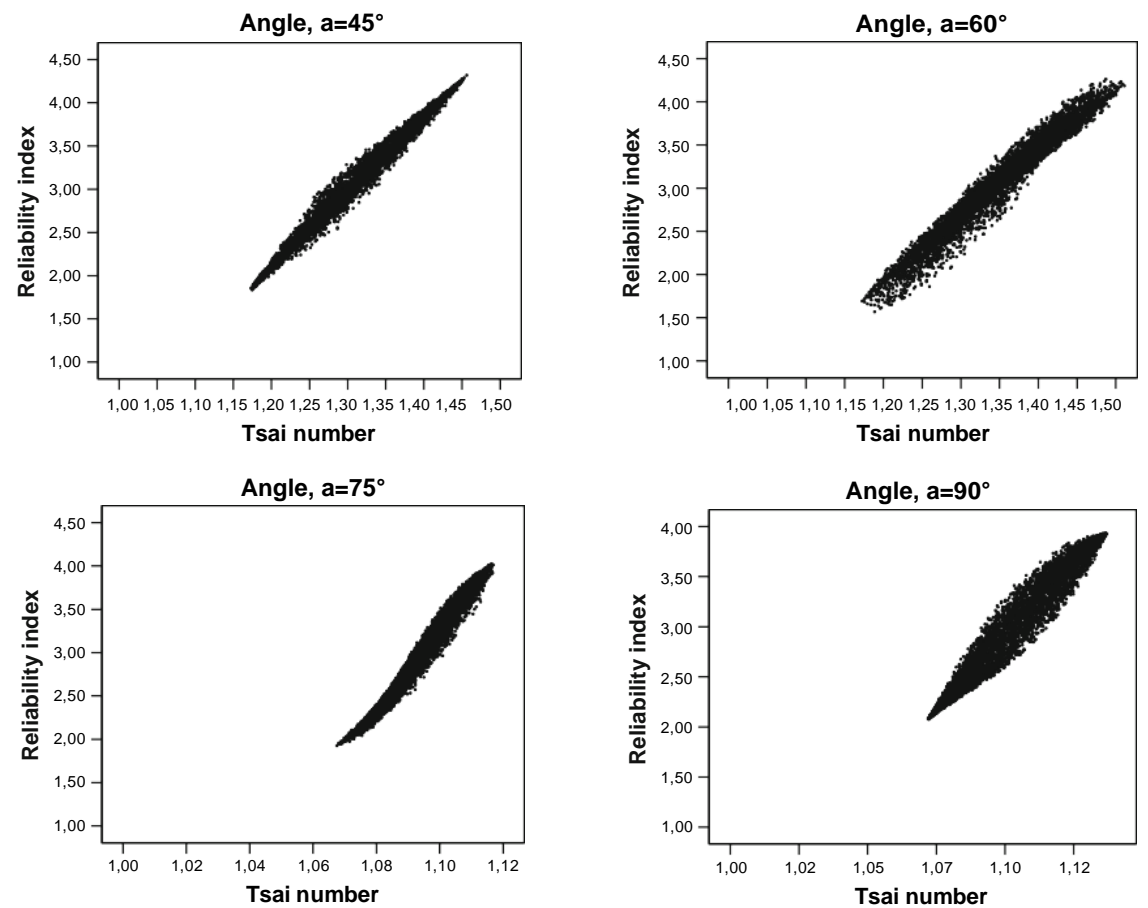

Fig. 6 Reliability index of the structure as a function of most critical Tsai number using ANN-based MCS 


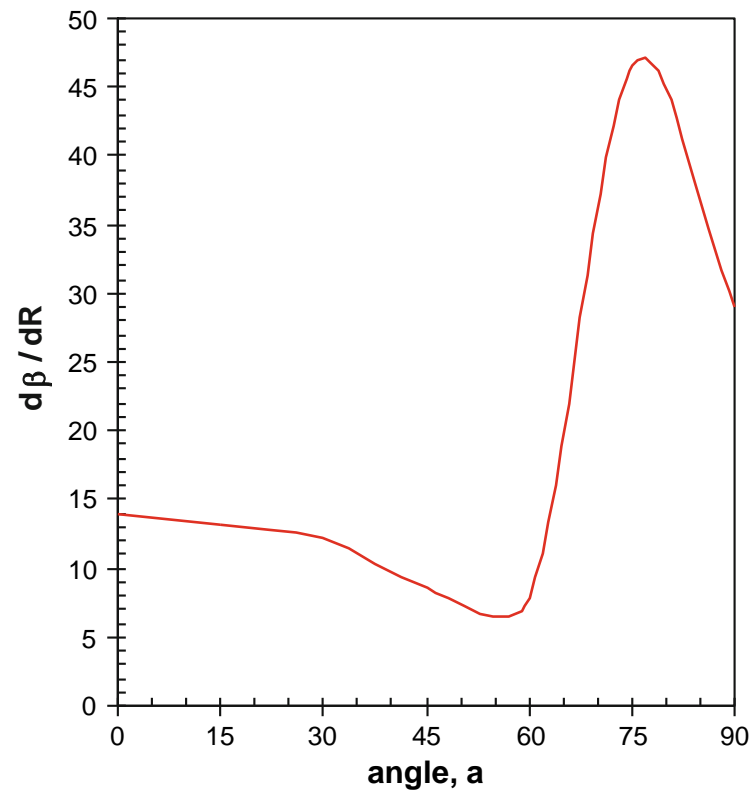

Fig. 7 Sensitivity of reliability index relative to the most critical Tsai number using ANN-based MCS

developed ANN, 5,000 simulations are generated for a uniform distribution of random variables $\pi=$ $\left[E_{1}, E_{2}, Y, S\right]$ and interval $\left[\bar{\pi}_{i}-0.06 \bar{\pi}_{i}, \bar{\pi}_{\mathrm{i}}+0.06 \bar{\pi}_{\mathrm{i}}\right]$, centered at the mean reference values $\bar{\pi}_{\mathrm{i}}$ defined in

Table 1. With these simulations, the relationship between the most critical Tsai number, $R$, and corresponding reliability index of the structure, $b_{\mathrm{s}}$, is established for different values of angle, $a$, as shown in Fig. 6.

From ANN-based MCS data, a linear regression based on the least square method is used to determine the best line fit of the simulated data presented in Table 4. The given coefficient of determination is the proportion of the total source of variation in the reliability index explained by the fit linear regression. The relationship between the reliability index and Tsai number is linear for most of the data generated by the ANN-based MCS approach.

The slope of the lines defined in Table 4 is the derivative $\frac{\partial \beta_{3}}{\partial R}$. Figure 7 demonstrates the dependence of the angle-ply $[-a /+a /-a /+a]_{s}$ composite laminate on the angle $a$. The derivative of the structural reliability index can then be calculated as follows:

$\frac{\partial \beta_{\mathrm{s}}}{\partial \pi_{\mathrm{i}}}=\frac{\partial \beta_{\mathrm{s}}}{\partial \bar{R}} \frac{\partial \bar{R}}{\partial \pi_{\mathrm{i}}}$

where $\frac{\partial \bar{R}}{\partial \pi_{i}}$ is an output of the developed ANN.

The objective of this section is to analyze the influence of mean reference value deviations on the structural response measured by the reliability index
Fig. 8 Interval of variation for relative sensitivities of the reliability index, $b_{\mathrm{s}}$, using ANN-based MCS
Relative sensitivities of the reliability index

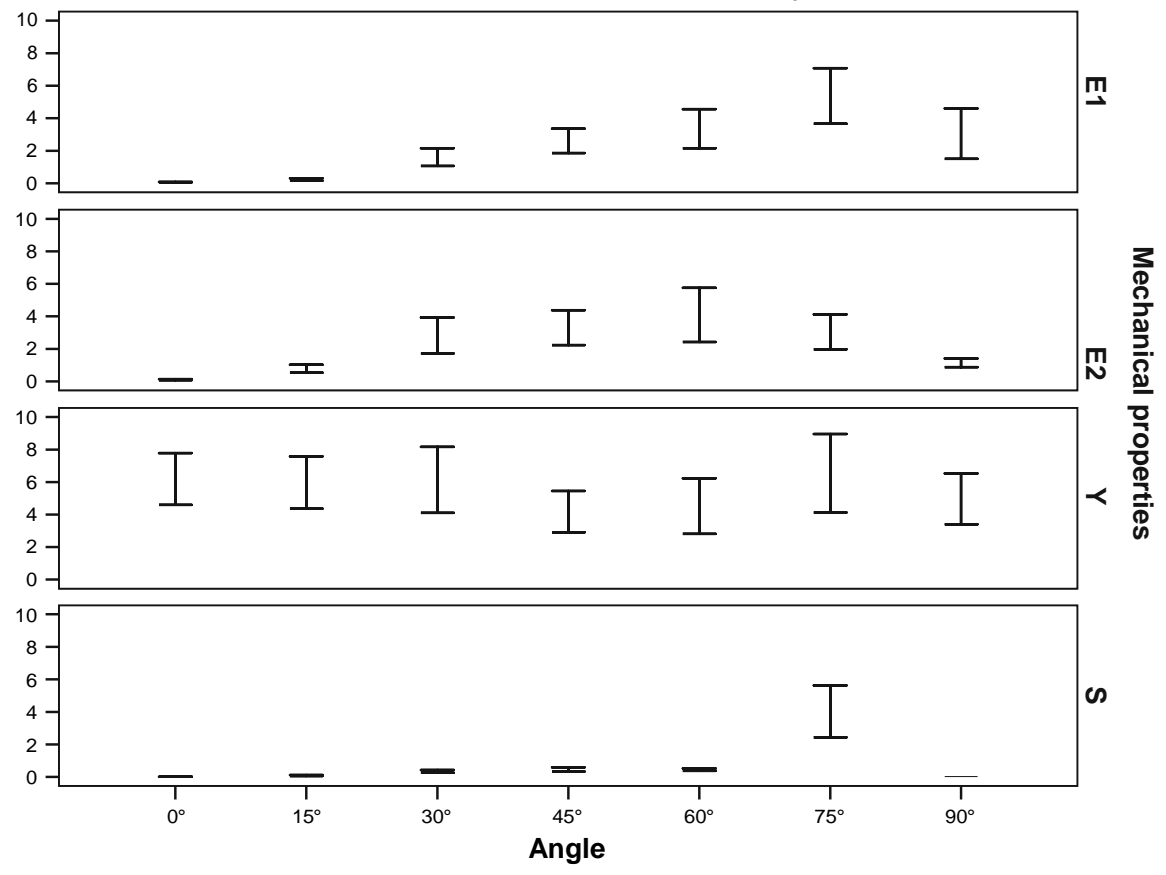


of the structure, $b_{\mathrm{s}}$. The concept of relative sensitivity, referred in Cacuci (2003), is applied to the reliability index of the structure and defined as

$\frac{\partial \beta_{s}^{*}}{\partial \pi_{i}}=\left|\frac{\partial \beta_{s}}{\partial \pi_{i}}\right|\left|\frac{\pi_{i}}{\beta_{s}}\right|$

where ${ }^{\mathrm{o} b_{\mathrm{i}}}{ }_{\mathrm{o}}$ is obtained from Eq. 16. The results for $b_{\mathrm{s}}$ are obtained from the ANN-based MCS approach for 5000 simulations, using previously defined values for $\left.p_{\mathrm{i}} 2 k_{\mathrm{i}}-0: 06 p_{\mathrm{i}} ; p_{\mathrm{i}} \mathrm{p} 0: 06 p_{\mathrm{i}}\right]$. Figure 8 shows the interval of variation for the relative sensitivities obtained from Eq. 17. The objective is to compare the relative importance of the input parameters on structural response, in particular for the inverse RBDO solutions. The reliability index, $b_{\mathrm{s}}$, is very sensitive to transverse strength, $Y$, over the entire domain of angle $a$. Considering the longitudinal elastic modulus $E_{1}$, and transverse elastic modulus $E_{2}$, the reliability index of the structure shows important variations over the intervals $a 2$ $1245^{0} ; 90^{\circ} \mathrm{J}$ and $a 2 k 30^{\circ} ; 75^{\circ} \mathrm{J}$, respectively. The sensitivity relative to the shear strength, $S$, is fair over the entire domain except for a 750 angle.

Analyzing the same 5,000 simulations of the ANN-based MCS, frequency histograms of the reliability index of the structure along the domain of angle $a$ are obtained and presented in Fig. 9. The histograms suggest a variation in the shape of the distribution of the reliability index for different angles. However, there are no important changes in the localization and dispersion of the results. Symmetry in the distribution and inexistence of the gaps is also verified for all angles.

For an angle, $a$, of 450 and 600, the data seem to be normally distributed, but the Kolmogorov-Smirnov test yields very low $p$-values, indicating significant results. Therefore, we conclude here that the data are not normally distributed for all angles.

Calculated descriptive statistics of the reliability index are presented in Table 5. The difference between maximum and minimum values is the most important aspect observed in Table 5. The quartiles of each distribution are clearly shown using an alternative box plot graph of the data, as shown in Fig. 10. The lower and upper quartiles define the box limits, and the median is near the middle of the box.

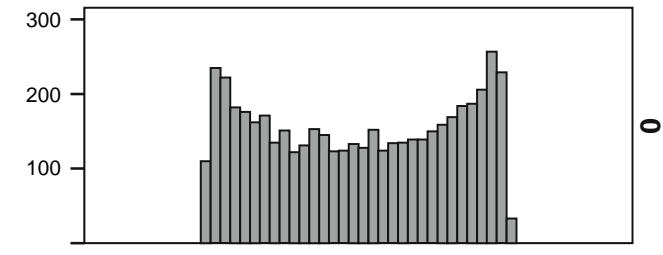

0
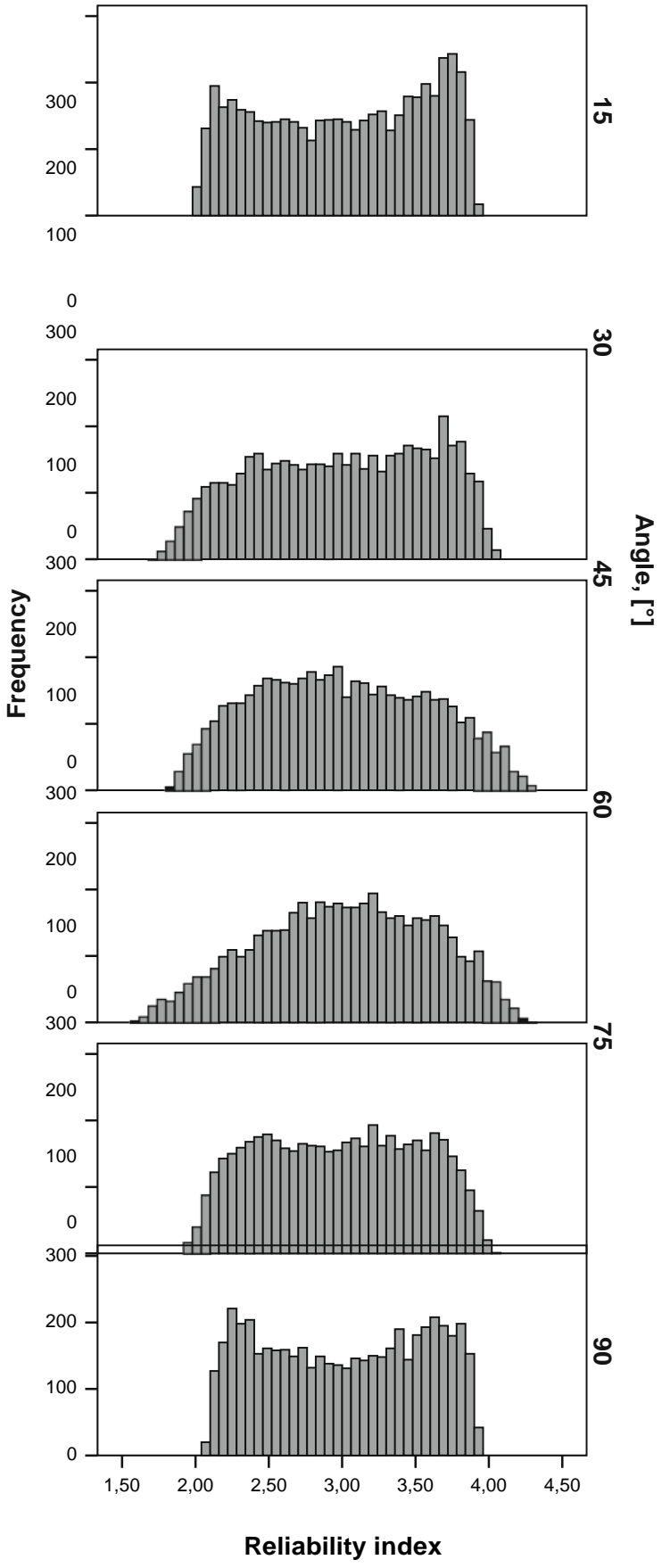

Fig. 9 Frequency histograms of the reliability index, $b_{\mathrm{s}}$, using data from the ANN-based MCS approach 
Table 5 Descriptive statistic of the reliability index, $b_{\mathrm{s}}$, using data from the ANN-based MCS approach

\begin{tabular}{lllll}
\hline Angle, $a(0)$ & Minimum & Maximum & Mean & SD \\
\hline 0 & 2.0468 & 3.9295 & 3.0010 & 0.5784 \\
15 & 1.9820 & 3.9295 & 3.0103 & 0.5665 \\
30 & 1.7233 & 4.0695 & 3.0009 & 0.5884 \\
45 & 1.8357 & 4.3191 & 3.0064 & 0.5825 \\
60 & 1.5698 & 4.2623 & 3.0107 & 0.5807 \\
75 & 1.9262 & 4.0268 & 2.9822 & 0.5305 \\
90 & 2.0797 & 3.9336 & 3.0156 & 0.5429 \\
\hline
\end{tabular}

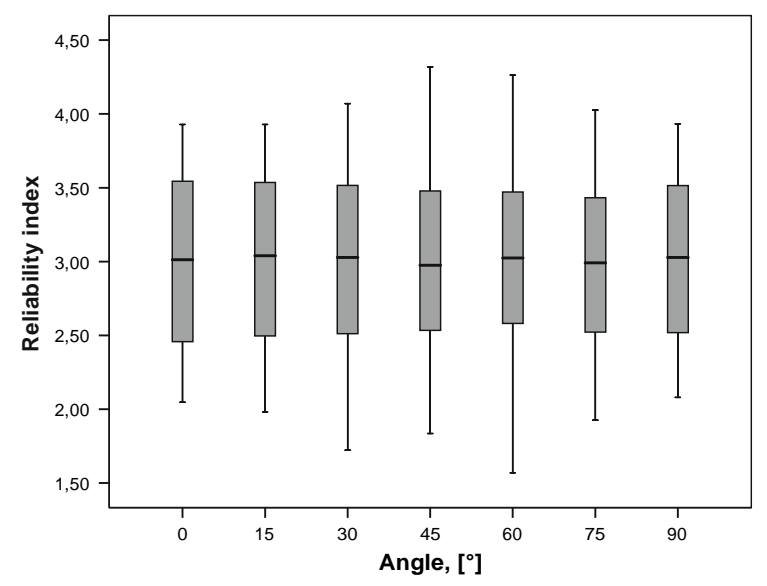

Fig. 10 Box plot of the reliability index, $b_{\mathrm{s}}$, using data from the ANN-based MCS approach

The range of each distribution is shown by the extended line. The box plot suggests that the median and inter-quartile ranges are similar for all angles, but the range is larger for 300, 450 and 600 angles. Symmetric distributions are observed for all angles.

From the previous analysis, it can be concluded that:

- The reliability index follows a non-normal and non-uniform probability distribution function.

- The mean value of the reliability index is close to 3 , as expected for all ply angles. This aspect confirms the accuracy of the proposed ANNbased MCS approach.

- The coefficient of variation of the reliability index is equal to $17 \%$. This value is very high when compared to the coefficient of variation for the input random variables, which has a predefined value of $6 \%$ of the mean values.
- The variability of the reliability index in RBDO and associated sensitivity must be considered for robust design of composite structures.

\section{Conclusions}

The influence of uncertainties of input parameters on the output response of composite structures is investigated in this paper. In particular, the effects of mechanical property deviations from the RBDO results are studied. The proposed ANN-based MCS approach shows that variations in the mean values of mechanical properties propagate and are even amplified in reliability index results in RBDO of composite structures. Based on the numerical results, the importance of measuring input parameters on structural response are established and discussed as a function of the anisotropy of composite materials. The efficiency of the proposed ANN-based MCS approach for uncertainty propagation analysis in RBDO has been demonstrated.

The study proves that the variability of the reliability index in RBDO, as a function of uncertainty of the mean values, can be very high. This high variability is also corroborated by evaluated relative sensitivity measures. These aspects must be considered for robust design since high structural response variability may induce a drastic reduction in the quality of the optimal design solutions for composite structures. Furthermore, other aspects related to the standard deviation and density probability distribution of the mechanical properties should also be considered in the analysis.

Acknowledgements The authors acknowledge "FCTFundação para a Ciência e Tecnologia" of Portugal for the financial support through research unit UMNMEE (10/225).

\section{References}

Adali, S., Lene, F., Duvaut, G., Chiaruttini, V.: Optimization of laminated composites subject to uncertain buckling loads. Compos. Struct. 62, 261-269 (2003)

Ahmad, S.: Curved finite elements in the analysis of solid, shell and plate structures. Ph.D. thesis, University College of Swansea, UK (1969)

António, C.C.: Optimization of structures using composite materials made of polymeric matrix. Ph.D. thesis, Faculty of Engineering, University of Porto, Portugal (1995) 
António, C.C.: A hierarchical genetic algorithm for reliability based design of geometrically non-linear composite structures. Compos. Struct. 54, 37-47 (2001)

António, C.C., Hoffbauer, L.N.: An approach for reliabilitybased robust design optimisation of angle-ply composites. Compos. Struct. 90, 53-59 (2009)

António, C.C., Torres Marques, A., Gonçalves, J.F.: Reliability based design with a degradation model of laminated composite structures. Struct. Optimizat. 12, 16-28 (1996)

Boyer, C., Béakou, A., Lemaire, M.: Design of a composite structure to achieve a specified reliability level. Reliab. Eng. Syst. Safety 56, 273-283 (1997)

Cacuci, D.G.: Sensitivity and Uncertainty Analysis, Vol. 1: Theory. Chapman \& Hall/CTC Press, Boca Raton, FL (2003)

Carbillet, S., Richard, F., Boubakar, L.: Reliability indicator for layered composites with strongly non-linear behaviour. Compos. Sci. Technol. 69, 81-87 (2009)

Cheng, J.: Hybrid genetic algorithms for structural reliability analysis. Comput. Struct. 85, 1524-1533 (2007)

Cheng, J., Li, Q.-S., Xiao, R.-C.: A new artificial neural network-based response surface method for structural reliability analysis. Probabilistic Eng. Mech. 23, 51-63 (2008)

Deng, J., Gu, D., Li, X., Yue, Z.Q.: Structural reliability analysis for implicit performance functions using artificial neural networks. Struct. Saf. 27, 25-48 (2005)

Fang, L., Wang, Y.: Number-Theoretic Methods in Statistics. CRC Press, FL, USA (1994)

Fang, K.-T., Wang, Y., Bentler, P.M.: Some applications of number-theoretic methods in statistics. Statistical Sci. 9(3), 416-428 (1994)
Figueiras, J.A.: Ultimate load analysis of anisotropic and reinforced concrete plates and shells. Ph.D. thesis, University College of Swansea, UK (1983)

Hasofer, A.M., Lind, N.C.: Exact and invariant second-moment code format. J. Eng. Mech. Div. ASCE 100, 111-121 (1974)

Liang, Y.-Z., Fang, K.-T., Xu, Q.-S.: Uniform design and its applications in chemistry and chemical engineering. Chemometr. Intell. Lab Syst. 58, 43-57 (2001)

Melchers, R.E.: Structural Reliability Analysis and Prediction. Wiley, Chichester (1999)

Nguyen-Thien, T., Tran-Cong, T.: Approximation of functions and their derivatives: a neural network implementation with applications. Appl. Math. Model. 23, 687-704 (1999) RaisRohani, M., Singh, M.N.: Comparison of global and local response surface techniques in reliability-based optimi- zation of composite structures. Struct. Multidiscipl. Optimizat. 26, 333-345 (2004)

Salas, P., Venkataraman, S.: Laminate optimization incorporating analysis and model parameter uncertainties for predictable failure. Struct. Multidiscipl. Optimizat. 37, 541-555 (2009)

Teters, G.A., Kregers, A.F.: Compromise optimization of a composite plate with a given probability of realization. Mech. Compos. Mater. 33(5), 441-448 (1997)

Tsai, S.W.: Composites Design. Think Composites, Dayton, USA (1987)

Zhang, L., Liang, Y.-Z., Jiang, J.-H., Yu, R.-Q., Fang, K.-T.: Uniform design applied to nonlinear multivariate calibration by ANN. Anal. Chim. Acta 370, 65-77 (1998) 